\title{
OCCURRENCE OF CARBAPENEMASES AND EXTENDED-SPECTRUM BETA-LACTAMASES IN UROPATHOGENIC ENTEROBACTERIACEAE ISOLATED FROM A COMMUNITY SETTING, SETTAT, MOROCCO
}

\author{
SAMIRA NATOUBI ${ }^{1,2 *}$, ABOUDDIHAJ BARGUIGUA ${ }^{3}$, NEZHA BAGHDAD ${ }^{2}$, KAOTAR NAYME $^{4}$, \\ MOHAMMED TIMINOUNI ${ }^{4}$, ABDERRAOUF HILALI ${ }^{1}$, SOUAD AMGHAR ${ }^{5}$, KHALID ZEROUALI $^{6,7}$
}

\begin{abstract}
${ }^{1}$ Department of Biology, Faculty of Science and Technology, Agrofood and Health Laboratory, University Hassan I, 26000, Settat, Morocco. ${ }^{2}$ Department of Microbiology, Microbiology Laboratory, Hassan II Hospital, 26000, Settat, Morocco. ${ }^{3}$ Department of Biology and Geology, Polyvalent Laboratory of Research and Development, Faculty Polydisciplinary, Sultan Moulay Slimane University, Bd Ibn Khaldoun, 23000, Beni Mellal, Morocco. ${ }^{4}$ Department of Research, Molecular Bacteriology Laboratory, Pasteur Institute of Morocco, 1 Place Louis Pasteur, 20360, Casablanca, Morocco. ${ }^{5}$ Department of Biology, Laboratory of Improved Soil Productivity and Environment, University Mohammed V, Higher Normal School, B.P: 5118, Rabat, Morocco. ${ }^{6}$ Department of Biomedical Sciences, Faculty of Medicine and Pharmacy, University Hassan II, Casablanca, Morocco. ${ }^{7}$ Department of Biology, Microbiology Laboratory, University Hospital Center, Ibn Rochd, 1 Street Hospital, 20360, Casablanca, Morocco. Email: samiranatoubi@yahoo.fr
\end{abstract}

Received: 27 August 2016, Revised and Accepted: 04 October 2016

\section{ABSTRACT}

Objective: Urinary tract infections (UTIs) are still commonly diagnosed in outpatients as well as in hospitalized patients. In this study, we investigated the prevalence and performed molecular characterization of extended-spectrum- $\beta$-lactamases (ESBL) and carbapenemases produced by Enterobacteriaceae isolates that cause community UTIs in Settat city, Morocco.

Methods: From January 2012 to December 2013, all uropathogenic community Enterobacteriaceae isolates were collected from the microbiology laboratory of Hassan II Hospital, Settat, Morocco. Antibiotic susceptibility testing was performed as recommended by Clinical and Laboratory Standard Institute. Phenotypic identification of ESBL and carbapenemase producer isolates was confirmed by the double-disk synergy test and the modified Hodge test, respectively. Molecular characterization of $\beta$-lactamase genes was performed using polymerase chain reaction (PCR), followed by sequencing of the obtained products.

Results: Among 153 isolates, 31 (20.26\%) were multi-drug resistant (MDR). Nine strains (5.88\%) were ESBL producers, of which Klebsiella pneumoniae ( $\mathrm{n}=5 ; 20.83 \%)$, Escherichia coli $(\mathrm{n}=3 ; 3.15 \%)$, and Enterobacter cloacae $(\mathrm{n}=1 ; 9.09 \%)$ species were identified. The results of ESBLencoding gene detection by sequencing revealed the presence of CTX-M-15 $(n=9)$ in association with other $\beta$-lactamase genes such as temoneira 1 $(\mathrm{n}=8)$ and sulfhydryl variable $1(\mathrm{n}=5)$. According to the modified Hodge test and PCR, three isolates $(1.96 \%)$ were positive carry the bla $a_{\mathrm{OXA}-48}$ gene.

Conclusion: The emergence of MDR uropathogenic Enterobacteriaceae isolates in our community is highly alarming. Strict measures will be required to control the further spread of these uropathogenic isolates.

Keywords: Carbapenemase, Enterobacteriaceae, Extended-spectrum- $\beta$-lactamases, Moroccan community.

(C) 2017 The Authors. Published by Innovare Academic Sciences Pvt Ltd. This is an open access article under the CC BY license (http://creativecommons, org/licenses/by/4. 0/) DOI: http://dx.doi.org/10.22159/ajpcr.2017.v10i1.14924

\section{INTRODUCTION}

Urinary tract infections (UTIs) are common bacterial infections that are still observed in outpatients and hospitalized patients [1]. They are the second most common infection appearing in the community [2]. Enterobacteriaceae are known to be the most common cause of UTIs [1]. Indeed, Escherichia coli and Klebsiella pneumoniae isolates are pathogens responsible for causing serious infections, both in the community and in health-care settings [3]. The past decade has shown an emergence of carbapenemase and extended-spectrum $\beta$-lactamase (ESBL) producing Enterobacteriaceae isolates in the community setting, the ESBLs being frequently plasmid encoded. These plasmids also carry genes encoding resistance to other antibiotics [4]. The majority of ESBL types are derived from temoneira (TEM) and sulfhydryl variable (SHV) genes by mutations. This extends the spectrum of beta-lactam antibiotics susceptible to hydrolysis by these enzymes are susceptible to hydrolysis by extended-spectrum antibiotics [4]. More than 180 TEMtype and 130 SHV-type $\beta$-lactamases had been identified worldwide (www.lahey.org/Studies/). More recently, CTX-M enzymes that preferentially hydrolyze and confer resistance to cefotaxime have been found in several continents around the world. More than 100 CTX-M type $\beta$-lactamases have been identified (www.lahey.org/Studies/), divided into five groups (CTX-M-1, 2, 8, 9 and 25).

In Moroccan hospitals, E. coli and K. pneumoniae isolate producing carbapenemase and ESBL have been the most predominant $[5,6]$. The $\beta$-lactamase genes detected in Moroccan hospital isolates were $b l a_{\text {стх-м }}$ $b l a_{\mathrm{TEM}}, b l a_{\mathrm{SHV}^{\prime}}, b l a_{\mathrm{DHA}}$ and bla $a_{\mathrm{OXA}}$ types $[5,7,8]$. Carbapenemase OXA-48 and NDM-1 producing Enterobacter cloacae, specifically K. pneumoniae, have been recently reported in Moroccan hospitals [5,7]. In addition, the occurrence of an OXA-48 producer in the environment has also been reported in Morocco [9].

Our study is known to be the first in Settat region, the objective of this study was to report the prevalence and characteristics of ESBL and carbapenemase-producing Enterobacteriaceae isolated from community acquired UTIs (CA-UTIs) in the Moroccan community of Settat city. It remains then, highly necessary to apply strict measures and adopt preventive strategies and continuous surveillance, to investigate the epidemiology of these multidrug-resistant organisms in the community. 


\section{METHODS}

\section{Setting and bacterial isolates}

From January 2012 to December 2013, urine specimens were collected from outpatients clinically suspected to have UTI, who had not previously been hospitalized. The urine specimens were analyzed at the microbiology laboratory at Hassan II Hospital, Settat. All urinary specimens with significant bacteremia ( $\geq 10^{5}$ colony-forming unit $/ \mathrm{mL}$ ) were further processed. The isolates were identified by conventional methods and biochemical tests using the Api20E collection (Biomerieux, Marcy l'Etoile, France).

The gender and age of the patients were recorded. Enterobacteriaceae isolated from currently or previously hospitalized patients were excluded from the study.

\section{Antimicrobial susceptibility testing}

Antimicrobial drug susceptibility testing of Enterobacteriaceae isolates was performed according to the disk diffusion method on MuellerHinton agar plates (Bio-Rad, Marnes-la-Coquette, France), and the results were interpreted according to the recommendations by the Clinical and Laboratory Standards Institute (CLSI) [10]. The following antimicrobial agents (Bio-Rad, Marnes-la-Coquette, France) were tested: Amoxicillin/clavulanic acid $(20 / 10 \mu \mathrm{g})$, cephalothin $(30 \mu \mathrm{g})$ cefoxitin $(30 \mu \mathrm{g})$, cefotaxime $(30 \mu \mathrm{g})$, ceftazidime $(30 \mu \mathrm{g})$, ceftriaxone $(30 \mu \mathrm{g})$, aztreonam $(30 \mu \mathrm{g})$, ciprofloxacin $(5 \mu \mathrm{g})$, gentamicin $(10 \mu \mathrm{g})$, amikacin $(30 \mu \mathrm{g})$, trimethoprim/sulfamethoxazole $(1.25 / 23.75 \mu \mathrm{g})$, imipenem $(10 \mu \mathrm{g})$, and ertapenem $(10 \mu \mathrm{g})$.

Multi-drug resistant Enterobacteriaceae (MDRE) were defined as isolates that were non-susceptible to at least one agent in three or more antimicrobial categories [11]. Isolates that did not meet the criteria were described as non-MDRE.

\section{Phenotypic detection of ESBL production}

ESBL production was screened using a double-disk synergy test between cefotaxime, ceftazidime, and aztreonam disks, placed at a distance of $30 \mathrm{~mm}$ (center to center) from the amoxicillin/clavulanic disk as previously described [12]. This test is considered as positive if a zone of inhibition is seen between the cephalosporin antibiotics and the amoxicillin/clavulanic disk, after $24 \mathrm{hrs}$ of incubation. The standard strains E. coli ATCC 25922 and K. pneumoniae ATCC 700603 were used as a negative and a positive control for ESBL production.

\section{Screening for carbapenemase production}

All isolates showing reduced susceptibility to an imipenem disk (diameter of zone of inhibition $<22 \mathrm{~mm}$ ) or an ertapenem disk (diameter of zone of inhibition $\leq 21 \mathrm{~mm}$ ) were screened for the production of carbapenemase according to the recommendations in the CLSI guidelines [10].

\section{Phenotypic detection of carbapenemase}

Phenotypic carbapenemase production was assessed using the modified Hodge test and interpreted according to the recommendations of the CLSI [10].

The phenotypic detection method for class B metallo- $\beta$-lactamase (MBL) production was performed using the combined disk test using two imipenem disks $(10 \mu \mathrm{g})$, one containing $10 \mu \mathrm{L}$ of $0.1 \mathrm{M}(292 \mu \mathrm{g})$ anhydrous ethylene diamine tetra-acetic acid (EDTA) (Sigma Chemicals, St. Louis, MO), placed $25 \mathrm{~mm}$ apart (center to center). MBL production was considered positive if the difference in diameter between the inhibition zones with an imipenem disk supplemented with EDTA and an imipenem disk alone was $>4 \mathrm{~mm}[12,13]$.

\section{Preparation of DNA template for PCR}

Total DNA was extracted by the boiling method by suspending a few colonies of an overnight culture of Enterobacteriaceae isolates growing on Luria-Bertani agar (Bio-Rad, Marnes-la-Coquette, France) in $500 \mu \mathrm{L}$ of DNase- and RNase-free water (Invitrogen, Paisley, UK) and boiling at $100^{\circ} \mathrm{C}$ for 10 minutes in a thermal block (Polystat 5, Bioblock Scientific, France), followed by centrifugation at $19,000 \times g$ for 5 minutes. An aliquot of $1 \mu \mathrm{L}$ of the supernatant was used as the DNA template for PCR.

\section{Detection of $\beta$-lactamase-encoding genes}

Enterobacteriaceae isolates were screened by PCR for the following $\beta$-lactamase-encoding genes: $b l a_{\text {стх-м }}$ of phylogenetic lineage groups 1,2 and 9, $b l a_{\mathrm{TEM}^{\prime}}, b l a_{\mathrm{SHV}}, b l a_{\mathrm{PER}}$, and bla $_{\mathrm{VEB}}$ genes as described previously [6]. Carbapenem-resistant Enterobacteriaceae were tested by using specific primers for carabapenem-hydrolysing enzymes encoding the genes $b l a_{\mathrm{GES}^{\prime}}, b l a_{\mathrm{KPC}}, b l a_{\mathrm{VIM}}, b l a_{\mathrm{IMP}}, b l a_{\mathrm{OXA}-48}$, and $b l a_{\mathrm{NDM}}$ as described previously [6].

Amplification reactions were performed in a volume of $50 \mu \mathrm{L}$ containing $1 \mu \mathrm{L}$ of DNA template, $2.5 \mathrm{mM} \mathrm{MgCl}, 0.4 \mu \mathrm{M}$ each of the forward and reverse primers, $100 \mu \mathrm{M}$ of each $\mathrm{dNTP}$, and 2 units of Taq DNA polymerase (Promega, Madison, USA) in $\times 1$ PCR buffer as provided by the manufacturer.

The cycling parameters were: 5 minutes of denaturation at $95^{\circ} \mathrm{C}$; followed by 30 cycles of denaturation $\left(95^{\circ} \mathrm{C}\right.$ for 1 minute), annealing $\left(52^{\circ} \mathrm{C}\right.$ for 1 minute for TEM and IMP; $58^{\circ} \mathrm{C}$ for 1 minute for NDM; $60^{\circ} \mathrm{C}$ for 1 minute for CTX-M, KPC, VIM, and SHV), and extension $\left(72^{\circ} \mathrm{C}\right.$ for 1 minute); ending with a final extension period of $72^{\circ} \mathrm{C}$ for 7 minutes.

PCR products were detected by electrophoresis on a $1.5 \%$ agarose gel (FMC Bioproduct, Rockland, USA) containing $1 \mu \mathrm{g} / \mathrm{mL}$ of ethidium bromide and analyzed under ultraviolet light. The gel picture was captured with an Olympus Digital Camera and analyzed using Digi-Docit software (UVP, Upland, USA).

\section{Sequencing of resistance genes}

All obtained amplification products were sequenced to validate their identities. Both strands of the purified amplicons were sequenced with a Genetic Analyzer $3130 \times 1$ sequencer (Applied Biosystems, Foster City, CA, USA), with the same primers used for PCR amplification. The nucleotide and deduced protein sequences were analyzed using online software at the National Center for Biotechnology Information website (www.ncbi.nlm.nih.gov).

\section{Statistical analysis}

The Pearson's $\chi^{2}$ or the Fisher's exact tests were used (SPSS version 20 software, IBM, Chicago, USA) for comparisons where appropriate. A $p<0.05$ was considered significant for all tests.

\section{RESULTS}

Among 1060 urine samples, 190 were positive for UTI, including $153(80.5 \%)$ that contained Enterobacteriaceae. Overall, 153 patients living in Settat city with Enterobacteriaceae-related CA-UTIs were recruited for this study. The strains were not duplicated. The mean patient age was 45 years (range 1-88 years, median 41 years), and the male/female ratio for these patients was 0.5 .

The distribution of the bacteria in outpatients with and without MDRE UTIs is shown in Table 1 . The majority of the uropathogens were E. coli $(62.09 \%)$, K. pneumoniae (15.69\%), Proteus mirabilis $(9.80 \%)$, and E. cloacae $(7.19 \%)$. E. coli was significantly more common in patients with non-MDRE UTIs than in those with MDRE UTIs $(66.39 \%$ versus $45.16 \%$; $\mathrm{p}=0.02$ ), whereas $K$. pneumoniae and E. cloacae were significantly a more common in patients with MDRE UTIs than in those with non-MDRE UTIs (Table 1).

Antibiotic resistance rates for Enterobacteriaceae isolates are shown in Table 2.

The overall resistance rates for amoxicillin/clavulanic acid, sulfamethoxazole-trimethoprim, ciprofloxacin, cefoxitin, and gentamicin were $59.48 \%, 47.7 \%, 24.18 \%, 22.88 \%$, and $18.30 \%$, respectively. $12(7.84 \%)$ strains were resistant to the third-generation cephalosporins. 
Seven strains (4.58\%) were immediately susceptible to ertapenem. Antibiotic resistance rates for MDRE UTIs were significantly greater than for non-MDRE UTIs for the majority of the tested antibiotics (Table 3).

ESBL production was detected in nine (5.88\%) Enterobacteriaceae isolates with a predominance of $K$. pneumoniae $(\mathrm{n}=5 ; 20.83 \%)$ followed by $E$. coli $(\mathrm{n}=3 ; 3.15 \%)$ and E. cloacae $(\mathrm{n}=1 ; 9.09 \%)$. The results of ESBL encoding gene detection by PCR revealed that the strains studied harbored a diversity of $\beta$-lactamases, namely CTX-M, SHV, and TEM
(Table 4). Further analysis of the $b l a_{\mathrm{TEM}}, b l a_{\mathrm{SHV}}$ and $b l a_{\mathrm{CTX}-\mathrm{M}}$ sequences identified the respective subgroups TEM-1 ( $\mathrm{n}=8)$, SHV-1 $(n=5)$ and CTX-M-15 (n=9). No amplicons were obtained for the other two tested CTX-M subgroups (Table 4), bla $a_{\mathrm{PER}}$ and $b l a_{\mathrm{VEB}}$ were not detected in any of the isolates. All combinations of bla genes detected are shown in Table 4. Among the nine $b l a_{\text {Стх-м-15 }}$ isolates, eight also harbored $b l a_{\text {TЕМ-1 }}$ and five harbored bla $_{\mathrm{SHV}}$ genes. It is interesting to note that the three $\beta$-lactamases genes bla $_{\mathrm{SHV}}$, bla $_{\mathrm{TEM}}$ and bla $a_{\mathrm{CTX}-\mathrm{M}}$ were coexpressed in four K. pneumoniae isolates.

Table 1: Distribution of Enterobacteriaceae isolates with and without multidrug resistance isolated from 153 study patients

\begin{tabular}{|c|c|c|c|c|c|c|}
\hline & n (\%) & & & & & \\
\hline Enterobacteriaceae Isolates & All isolates & MDR-isolates & Non-MDR isolates & $\mathbf{p}$ & OR & $95 \%$ CI \\
\hline K. pneumoniae & $24(15.69)$ & $9(29.03)$ & $15(12.30)$ & 0.02 & 2.91 & $1.13-7.50$ \\
\hline P. mirabilis & $15(9.80)$ & $1(3.23)$ & $14(11.48)$ & 0.19 & 0.24 & $0.03-1.96$ \\
\hline E. cloacae & $11(7.19)$ & $5(16.13)$ & $6(4.92)$ & 0.03 & 3.71 & $1.05-13.11$ \\
\hline C. freundii & $7(4.58)$ & $2(6.45)$ & $5(4.10)$ & 0.57 & 1.61 & $0.29-8.74$ \\
\hline K. oxytoca & $1(0.65)$ & 0 & $1(0.82)$ & - & - & - \\
\hline Total & $153(100)$ & $31(100)$ & $122(100)$ & - & - & - \\
\hline
\end{tabular}

MDR: Multidrug resistant, Non-MDR: Non-multidrug resistant, OR: Odds ratio, CI: Confidence interval, E. coli: Escherichia coli, K. pneumoniae: Klebsiella pneumoniae P. mirabilis: Proteus mirabilis, E. cloacae: Enterobacter cloacae, C. freundii: Citrobacter freundii, K. oxytoca: Klebsiella oxytoca

Table 2: Antibiotic resistance rates among Enterobacteriaceae species isolated from study patients

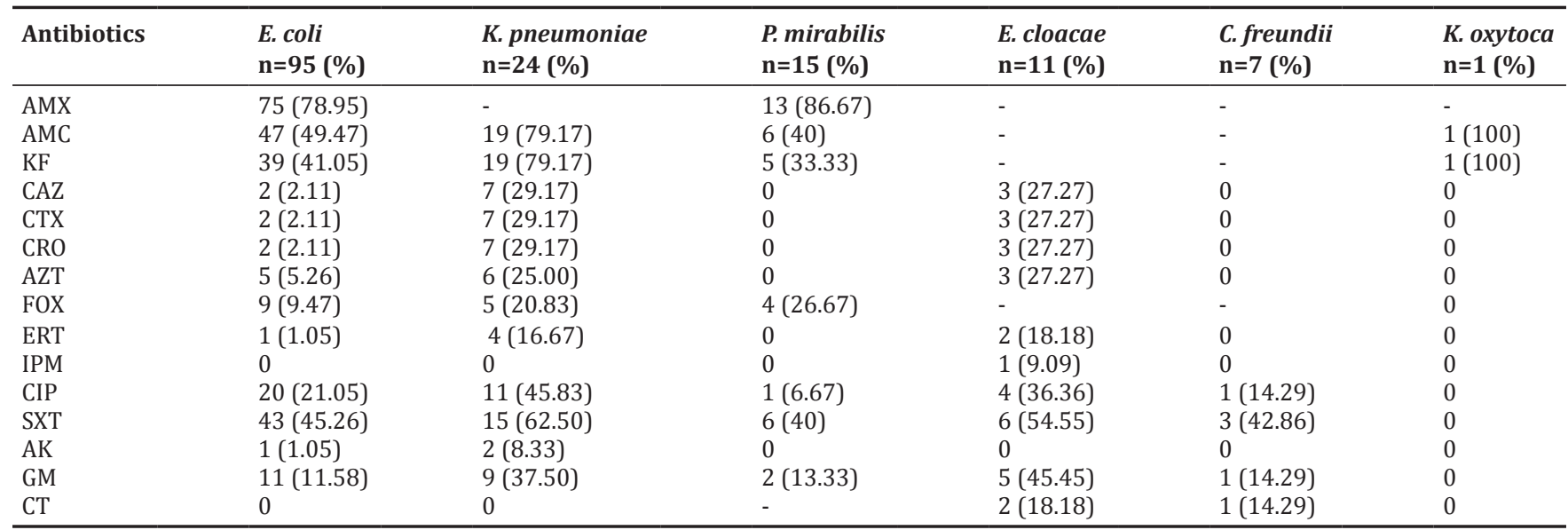

AMX: Amoxicillin, AMC: Amoxicillin/clavulanic acid, KF: Cephalothin, CTX: Cefotaxime, CRO: Ceftriaxone, CAZ: Ceftazidime, AZT: Aztreonam, FOX: Cefoxitin, ERT: Ertapenem, IPM: Imipenem, CIP: Ciprofloxacin, SXT: Sulfamethoxazole/trimethoprim, AK: Amikacin, GM: Gentamicin and CT: Colistin, E. coli: Escherichia coli, K. pneumoniae: Klebsiella pneumoniae, P. mirabilis: Proteus mirabilis, E. cloacae: Enterobacter cloacae, C. freundii: Citrobacter freundii, K. oxytoca: Klebsiella oxytoca

Table 3: Antibiotic resistance rates among 153 unique Enterobacteriaceae bacterial isolates from study patients, with and without MDR

\begin{tabular}{|c|c|c|c|c|c|c|}
\hline Antibiotics & All isolates n (\%) & MDR n (\%) & non-MDR n (\%) & $\mathbf{p}$ & OR & $95 \% \mathrm{CI}$ \\
\hline AMX & 131 (85.62) & 30 (96.77) & 101 (82.79) & 0.12 & 3.11 & $0.69-14.07$ \\
\hline AMC & $91(59.48)$ & 26 (83.87) & $65(53.28)$ & 0.001 & 4.56 & $1.64-12.65$ \\
\hline $\mathrm{KF}$ & 82 (53.59) & 26 (83.87) & $56(45.90)$ & 0.0003 & 5.10 & $1.96-13.29$ \\
\hline CAZ & $12(7.84)$ & 11 (35.48) & $1(0.82)$ & $>0.0001$ & 66.55 & $8.14-544.06$ \\
\hline CTX & $12(7.84)$ & 11 (35.48) & $1(0.82)$ & $>0.0001$ & 66.55 & $8.14-544.06$ \\
\hline CRO & $12(7.84)$ & 11 (35.48) & $1(0.82)$ & $>0.0001$ & 66.55 & $8.14-544.06$ \\
\hline AZT & $14(9.15)$ & $14(45.16)$ & 0 & $>0.0001$ & & \\
\hline ERT & $7(4.58)$ & $7(22.58)$ & 0 & $>0.0001$ & & \\
\hline IPM & $1(0.65)$ & $1(3.23)$ & 0 & 0.04 & & \\
\hline CIP & 37 (24.18) & $27(87.10)$ & $10(8.20)$ & $>0.0001$ & 75.60 & $22.02-259.51$ \\
\hline SXT & 73 (47.71) & 29 (93.55) & 44 (36.07) & $>0.0001$ & 25.70 & $5.85-112.89$ \\
\hline $\mathrm{AK}$ & $3(1.96)$ & $3(9.68)$ & 0 & 0.00006 & & \\
\hline GM & $28(18.30)$ & $19(61.29)$ & $9(7.38)$ & $>0.0001$ & 18.35 & $6.89-48.84$ \\
\hline CT & $3(1.96)$ & 0 & $3(2.46)$ & 1 & & \\
\hline
\end{tabular}

AMX: Amoxicillin, AMC: Amoxicillin/clavulanic acid, KF: Cephalothin, CTX: Cefotaxime, CRO: Ceftriaxone, CAZ: Ceftazidime, AZT: Aztreonam, FOX: Cefoxitin, ERT: Ertapenem, IPM: Imipenem, CIP: Ciprofloxacin, SXT: Sulfamethoxazole/trimethoprim, AK: Amikacin, GM: Gentamicin, CT: Colistin, MDR: Multidrug resistant, Non-MDR: Non-multidrug resistant, OR: Odds ratio, CI: Confidence interval 
Table 4: Characteristics of the ESBL-producing Enterobacteriaceae isolates collected from a Moroccan community

\begin{tabular}{lllllll}
\hline Code & Species & $\begin{array}{l}\text { Date of isolation } \\
\text { (DD/MM/YYY) }\end{array}$ & Gender/age & $\boldsymbol{\beta}$-lactamase & Carbapenemase type & $\begin{array}{l}\text { Antibiotic } \\
\text { resistance pattern* }\end{array}$ \\
\hline Ec1 & E. cloacae & $11 / 04 / 2012$ & M/65 & - & OXA-48 & FOX, ERT, SXT, CIP, GM \\
Ec2 & E. cloacae & $07 / 10 / 2013$ & M/60 & CTX-M-15, TEM-1 & & FOX, ERT, SXT, CIP, GM \\
E1 & E. coli & $04 / 04 / 2012$ & M/60 & CTX-M-15, TEM-1 & OXA-48 & FOX, SXT, GM \\
E2 & E. coli & $27 / 06 / 2012$ & M/60 & CTX-M-15, TEM-1 & & FOX, SXT, CIP \\
E3 & E. coli & $17 / 12 / 2013$ & M/65 & CTX-M-15, TEM-1 & OXA-48 & FOX, SXT, CIP, GM \\
K1 & K. pneumoniae & $16 / 01 / 2013$ & F/70 & & FOX, SXT, CIP \\
K2 & K. pneumoniae & $26 / 01 / 2012$ & M/64 & CTX-M-15, TEM-1, SHV-1 & & FOX, SXT, CIP, GM \\
K3 & K. pneumoniae & $01 / 06 / 2013$ & M/50 & CTX-M-15, TEM-1, SHV-1 & & SXT, CIP, GM \\
K4 & K. pneumoniae & $02 / 10 / 2013$ & M/65 & CTX-M-15, TEM-1, SHV-1 & & FOX, ERT, SXT, CIP, GM \\
K5 & K. pneumoniae & $09 / 11 / 2012$ & M/65 & CTX-M-15, TEM-1, SHV-1 & & SXT, CIP, GM \\
K6 & K. pneumoniae & $11 / 12 / 2012$ & M/66 & CTX-M-15, SHV-1 & SXT, CIP, GM \\
\hline
\end{tabular}

*All isolates were resistant to amoxicillin, amoxicillin/clavulanic acid, cephalothin, cefotaxime, ceftriaxone, ceftazidime and aztreonam. M: Male, F: Female,

FOX: Cefoxitin, ERT: Ertapenem, SXT: Sulfamethoxazole/trimethoprim, CIP: ciprofloxacin, GM: Gentamicin, E. coli: Escherichia coli, K. pneumoniae: Klebsiella pneumoniae, P. mirabilis: Proteus mirabilis, E. cloacae: Enterobacter cloacae, C. freundii: Citrobacter freundii, K. oxytoca: Klebsiella oxytoca, ESBL: Extended-spectrum- $\beta$-lactamases

Among seven carbapenem-resistant isolates, three $(3 / 153 ; 1.96 \%)$ were positive in the modified Hodge test and were identified as K. pneumoniae, E. coli, and E. cloacae. Only one carbapenemaseproducing isolate (E. coli E1) was an ESBL co-producer. The results of carbapenemase gene detection by PCR and sequencing revealed that the strains studied harbored the $b l a_{\mathrm{OXA}-48}$ gene (Table 4)

\section{DISCUSSION}

Carbapenemase and ESBL-producing Enterobacteriaceae have become a worldwide problem. Enterobacteriaceae have become one of the most important causes of nosocomial and communityacquired infections [14,15]. $\beta$-lactams (mainly extended-spectrum cephalosporins, carbapenems) and fluoroquinolones represent the main therapeutic option to treat infections due to these bacteria [2]. However, the extensive use of these antibiotics has caused both the emergence and the progressive spread of this health-threatening problem.

In Morocco, several studies demonstrate a wide dissemination of ESBLs in the environments, in both clinical and community settings $[8,16,17]$, especially in community-acquired UTIs $[16,18,19]$. Most urinary and intra-abdominal infections with E. coli and $K$. pneumoniae are endogenous [20]. The ESBL producing Enterobacteriaceae showed a high frequency of resistance to various antimicrobial agents ( $\beta$-lactams, ciprofloxacin, sulfamethoxazole trimethoprim, aztreonam) which have been reported increasingly worldwide [18,21]. E. coli and Klebsiella spp. producing $\beta$-lactamases play an important role in resistance to $\beta$-lactams [22]. The mechanism of resistance to the third-generation cephalosporins in non-ESBL producers could be due to the production of AmpC $\beta$-lactamase [23]

Our study reveals a higher prevalence of ESBL-producing K. pneumoniae (20.83\%) and ESBL-producing E. coli $(3.26 \%)$ in the Moroccan community than recorded in previous Moroccan reports [18].

This indicates that in a 2-year interval, the prevalence of ESBLs among urinary $K$. pneumoniae has been increasing, and shows that ESBL-producing strains continue to disseminate in Moroccan communities.

The inappropriate and extensive use of broad-spectrum antibiotics can lead to the emergence and spread of these ESBL producing strains. Another cause remains a person-to-person transmission via fecal carries between within families as reported by Rodriguez-Bano et al. [24]. Furthermore, evidence of ESBL-producing isolates in cattle, poultry, dogs, and cats suggests that food-producing animals and house pets might act as a reservoir for the transmission or acquisition of ESBL genes or ESBL-producing Enterobacteriaceae in the community $[25,26]$. Furthermore, antibiotics are commonly used in animals for prophylaxis or as performance enhancers, and such practices are likely to increase the development of resistance.
In general, the resistance patterns of ESBL-producing bacteria studied here were similar to those frequently described in various studies, i.e., the ESBL producers were resistants to $\beta$-lactams, fluoroquinolones, aminoglycosides and trimethoprim/sulfamethoxazole [18,26], which contributes to the selection and persistence of multidrug-resistant ESBL strains and plasmids in both clinical and community settings.

We detected a variety of $\beta$-lactamases among Enterobacteriaceae isolates, namely SHV-, CTX-M-, and TEM-type enzymes. The CTX-M type was the most common ESBL in our setting $[16,19]$. The predominance of CTX-M-15 indicates that this variant is now common in Morocco, as in other countries, as a result of worldwide dissemination $[27,28]$. The higher incidence of CTX-M among ESBL enzymes is probably associated with the mobilization and incorporation of bla $a_{\text {стхм }}$ into mobile genetic elements. Cantón et al. report that $b l a_{\text {СТX-M }}$ genes have been mobilized to plasmids almost ten times more frequently than other class A $\beta$-lactamases [29].

The coexistence of different $\beta$-lactamase genes within the same isolate as found in this study, has also been reported in other countries [30,31]. Although the clinical importance of ESBLs in patients carrying ESBLexpressing K. pneumoniae is clear, the clinical importance of non-ESBL enzymes is yet to be revealed. Among these non-ESBL enzymes, we identified SHV-1, an intrinsic gene in K. pneumoniae. Co-expression of CTX-M enzymes and SHV-1 penicillinases is most commonly found in K. pneumoniae isolates [32,33].

The OXA-48-producing Enterobacteriaceae isolates described in Moroccan communities and hospitals may have a relationship to those identified in France and the Netherlands [9].

This report illustrates the widespread of carbapenemase-producing Enterobacteriaceae in the Mediterranean area and in Europe. Our search showed trends of the emergence of carbapenem-resistant Enterobacteriaceae in Moroccan community settings along with the carriage of resistance genes with clinical relevance coexpressed by $b l a_{\text {OXA-48 }}[18]$. The spread of the same major carrying plasmid between Moroccan cities has been identified. This finding emphasizes the importance of systematic detection to contain the spread of these multidrug-resistant organisms.

\section{CONCLUSION}

Our study reported an increase in the prevalence of ESBL and OXA-48 carbapenemase-producing multidrug-resistant Enterobacteriaceae in Morocco. These data suggest that specific strategies for regular surveillance and control of antimicrobial resistance agents are required. Antibiotic resistance remains a serious issue, as the rapid spread of these bacteria would eventually limit clinical treatment choices. To avoid UTI treatment failures, it will be necessary to start monitoring ESBL and carbapenemase producers in laboratories using conventional antibiograms. 


\section{REFERENCES}

1. Caron F. Diagnosis and treatment of community-acquired urinary tract infections in adults: What has changed? Comments on the 2008 guidelines of the French Health Products Safety Agency (AFSSAPS). Presse Med 2010; 39(1): 42-8.

2. Thapa R, Lamichhane P, Banjara MR, Acharya GP. Prevalence of extended spectrum beta-lactamase producing uropathogens in pregnant women. Asian J Pharm Clin Res 2015;8:207-10.

3. Tansarli GS, Athanasiou S, Falagas ME. Evaluation of antimicrobial susceptibility of Enterobacteriaceae causing urinary tract infections in Africa. Antimicrob Agents Chemother 2013;57(8):3628-39.

4. Paterson DL, Bonomo RA. Extended-spectrum beta-lactamases: A clinical update. Clin Microbiol Rev 2005;18(4):657-86.

5. Barguigua A, Zerouali K, Katfy K, El Otmani F, Timinouni M, Elmdaghri N. Occurrence of OXA-48 and NDM-1 carbapenemaseproducing Klebsiella pneumoniae in a Moroccan university hospital in Casablanca, Morocco. Infect Genet Evol 2015;31:142-8.

6. Barguigua A, El Otmani F, Talmi M, Zerouali K, Timinouni M. Emergence of carbapenem-resistant Enterobacteriaceae isolates in the Moroccan community. Diagn Microbiol Infect Dis 2012;73(3):290-1.

7. Girlich D, Bouihat N, Poirel L, Benouda A, Nordmann P. High rate of faecal carriage of extended-spectrum $\beta$-lactamase and OXA-48 carbapenemase-producing Enterobacteriaceae at a University Hospital in Morocco. Clin Microbiol Infect 2013;4(10):1469-91.

8. Natoubi S, Barguigua A, Zriouil SB, Baghdad N, Timinouni M, Hilali A, et al. Incidence of extended-spectrum beta-lactamase-producing Klebsiella pneumoniae among patients and in the environment of Hassan II Hospital, Settat, Morocco. Adv Microbiol 2016;6:152-61.

9. Potron A, Poirel L, Bussy F, Nordmann P. Occurrence of the carbapenemhydrolyzing beta-lactamase gene blaOXA-48 in the environment in Morocco. Antimicrob Agents Chemother 2011;55(11):5413-4.

10. CLSI. Clinical and Laboratory Standards Institute. Performance Standards for Antimicrobial Susceptibility Testing: Twenty-first Informational Supplement M100-S21. Wayne, PA, USA: CLSI; 2011.

11. Magiorakos AP, Srinivasan A, Carey RB, Carmeli Y, Falagas ME, Giske CG, et al. Multidrug-resistant, extensively drug-resistant and pandrug-resistant bacteria: An international expert proposal for interim standard definitions for acquired resistance. Clin Microbiol Infect 2012;18(3):268-81

12. Garrec H, Drieux-Rouzet L, Golmard JL, Jarlier V, Robert J. Comparison of nine phenotypic methods for detection of extendedspectrum beta-lactamase production by Enterobacteriaceae. J Clin Microbiol 2011;49(3):1048-57.

13. Franklin C, Liolios L, Peleg AY. Phenotypic detection of carbapenemsusceptible metallo-beta-lactamase-producing gram-negative bacilli in the clinical laboratory. J Clin Microbiol 2006;44(9):3139-44.

14. Djahmi N, Dunyach-Remy C, Pantel A, Dekhil M, Sotto A, Lavigne JP. Epidemiology of carbapenemase-producing Enterobacteriaceae and Acinetobacter baumannii in Mediterranean countries. Biomed Res Int 2014;2014:305784.

15. Kung $\mathrm{CH}, \mathrm{Ku}$ WW, Lee $\mathrm{CH}$, Fung $\mathrm{CP}$, Kuo SC, Chen TL, et al. Epidemiology and risk factors of community-onset urinary tract infection caused by extended-spectrum $\beta$-lactamase-producing Enterobacteriaceae in a medical center in Taiwan: A prospective cohort study. J Microbiol Immunol Infect 2015;48(2):168-74

16. Barguigua A, Ouair H, El Otmani F, Saile R, El Mdaghri N, El Azhari M, et al. Fecal carriage of extended-spectrum ß-lactamase-producing Enterobacteriaceae in community setting in Casablanca. Infect Dis (Lond) $2015 ; 47(1): 27-32$.
17. Barguigua A, El Otmani F, Talmi M, Zerouali K, Timinouni M. Prevalence and types of extended spectrum ß-lactamases among urinary Escherichia coli isolates in Moroccan community. Microb Pathog 2013;61-62:16-22.

18. Barguigua A, El Otmani F, Talmi M, Bourjilat F, Haouzane F, Zerouali $\mathrm{K}$, et al. Characterization of extended-spectrum B-lactamaseproducing Escherichia coli and Klebsiella pneumoniae isolates from the community in Morocco. J Med Microbiol 2011;60:1344-52.

19. Barguigua A, El Otmani F, Talmi M, Reguig A, Jamali L, Zerouali K, et al. Prevalence and genotypic analysis of plasmid-mediated ß-lactamases among urinary Klebsiella pneumoniae isolates in Moroccan community. J Antibiot (Tokyo) 2013;66(1):11-6.

20. Søraas A, Sundsfjord A, Sandven I, Brunborg C, Jenum PA. Risk factors for community-acquired urinary tract infections caused by ESBL-producing Enterobacteriaceae - A case-control study in a low prevalence country. PLoS One 2013;8(7):e69581.

21. Minarini LA, Gales AC, Palazzo IC, Darini AL. Prevalence of community-occurring extended spectrum beta-lactamase-producing Enterobacteriaceae in Brazil. Curr Microbiol 2007;54(5):335-41.

22. Ramalingam AJ. Carbapenemases and gram-negative bacteria. Asian J Pharm Clin Res 2016;9(3):18-9.

23. Jacoby GA. AmpC beta-lactamases. Clin Microbiol Rey 2009;22(1):161-82.

24. Rodriguez-Bano J, Lopez-Cerero L, Navarro MD, Diaz de Alba P, Pascual A. Faecal carriage of extended-spectrum beta-lactamaseproducing Escherichia coli: Prevalence, risk factors and molecular epidemiology. J Antimicrob Chemother 2008;62(5):1142-9.

25. Carattoli A. Animal reservoirs for extended spectrum beta-lactamase producers. Clin Microbiol Infect 2008;14:117-23.

26. Moreno A, Bello H, Guggiana D, Domínguez M, González G. Extended-spectrum beta-lactamases belonging to CTX-M group produced by Escherichia coli strains isolated from companion animals treated with enrofloxacin. Vet Microbiol 2008;129(1-2):203-8.

27. Betitra Y, Teresa V, Miguel V, Abdelaziz T. Determinants of quinolone resistance in Escherichia coli causing community-acquired urinary tract infection in Bejaia, Algeria. Asian Pac J Trop Med 2014;7(6):462-7.

28. Baudry PJ, Nichol K, DeCorby M, Lagace-Wiens P, Olivier E, Boyd D, et al. Mechanisms of resistance and mobility among multidrug-resistant CTX-M-producing Escherichia coli from Canadian intensive care units: The $1^{\text {st }}$ Report of QepA in North America. Diagn Microbiol Infect Dis 2009;63(3):319-26

29. Cantón R, González-Alba JM, Galán JC. CTX-M enzymes: Origin and diffusion. Front Microbiol 2012;3:110.

30. Arpin C, Quentin C, Grobost F, Cambau E, Robert J, Dubois V, et al. Nationwide survey of extended-spectrum \{beta\}-lactamase-producing Enterobacteriaceae in the French community setting. J Antimicrob Chemother 2009;63(6):1205-14.

31. Woodford N, Carattoli A, Karisik E, Underwood A, Ellington MJ, Livermore DM. Complete nucleotide sequences of plasmids pEK204, pEK499, and pEK516, encoding CTX-M enzymes in three major Escherichia coli lineages from the United Kingdom, all belonging to the international O25:H4-ST131 clone. Antimicrob Agents Chemother 2009;53(10):4472-82.

32. Breurec S, Guessennd N, Timinouni M, Le TA, Cao V, Ngandjio A, et al. Klebsiella pneumoniae resistant to third-generation cephalosporins in five African and two Vietnamese major towns: Multiclonal population structure with two major international clonal groups, CG15 and CG258. Clin Microbiol Infect 2013;19(4):349-55.

33. Philippon A. Les bêta-lactamases à spectre élargi ou étendu (BLSE). Immuno Anal Biol Spec 2013;28(56):287-96. 